\title{
Optically induced structural phase transitions in ion Coulomb crystals
}

\author{
Peter Horak, ${ }^{1}$ Aurélien Dantan, ${ }^{2}$ and Michael Drewsen ${ }^{2}$ \\ ${ }^{1}$ Optoelectronics Research Centre, University of Southampton, Southampton SO17 1BJ, United Kingdom \\ ${ }^{2}$ QUANTOP, Danish National Research Foundation Center for Quantum Optics, \\ Department of Physics and Astronomy, University of Aarhus, DK-8000 Aarhus C, Denmark
}

(Dated: October 23, 2012)

\begin{abstract}
We investigate numerically the structural dynamics of ion Coulomb crystals confined in a threedimensional harmonic trap when influenced by an additional one-dimensional optically induced periodical potential. We demonstrate that transitions between thermally excited crystal structures, such as body-centered cubic and face-centered cubic, can be suppressed by a proper choice of the potential depth and periodicity. Furthermore, by varying the harmonic trap parameters and/or the optical potential in time, controlled transitions between crystal structures can be obtained with close to unit efficiency.
\end{abstract}

PACS numbers: 37.10.Ty, 37.10.Vz, 64.70.kp, 36.40.Ei

When an ensemble of confined ions with the same sign of charge is cooled to a sufficiently low temperature, the ionic system forms a crystalline structure [1], often referred to as an ion Coulomb crystal. Since the first experimental realizations of ion Coulomb crystals through laser cooling of atomic ions into the milli-Kelvin regime in electromagnetic traps $[2,3]$, there has been growing theoretical [4-14] and experimental [15-24] interest in studying the structural and dynamic properties of these crystals under different trapping conditions and for various ion compositions.

The unique localization and isolation of the individual ions constituting the crystals have already led to a large number of amazing results within precision measurements [25], cavity quantum electrodynamics (CQED) [26-30], quantum information science [31-35], and cold molecular science [36-39]. For experiments involving larger three-dimensional ion Coulomb crystals, such as CQED related experiments [26, 27] with the interesting prospect of creating quantum memories and other quantum devices, full structural control of the crystal structures is still in need for optimizing the coupling between the ions and the cavity modes.

While the energetic ground state of very large threedimensional Coulomb crystals ( $\gtrsim 10^{5}$ ions) in a harmonic confinement is known to be a body-centered cubic (bcc) lattice [1], the energetically most favorable configuration for smaller crystals ( $\lesssim 10^{3}$ ions) is ions situated in concentric shells [5]. For medium sized crystals often employed in experiments $[26,27]\left(\sim 10^{3}-10^{5}\right.$ ions $)$, the structure is generally not very stable, and thermally induced transitions between a large variety of states including metastable bcc and fcc structures and incommensurable crystallite formations can be observed [40, 41]. While structural stability can be dramatically increased using two-species crystals [21, 23], means to control and manipulate the structures of single-species crystals are highly wanted, not only for applications in quantum information science, but also for exploiting Coulomb crys- tals as simulators of solid state physics, like structural transitions of iron under extreme pressure [42] of relevance for geophysics and thin-film growth [43] of importance for nanotechnology.

In this Letter, we report on molecular dynamics (MD) simulations of harmonically trapped ion Coulomb crystals in the presence of an additional periodically corrugated potential in the form of an induced dipole potential originating from a far off-resonant standing-wave light field [44]. We demonstrate how such a potential can be exploited to prevent thermally induced crystal phase transitions and/or to induce controlled and efficient transitions between bcc and fcc crystal structures.

In our model an ensemble of $N$ trapped and laser cooled ions with positions $\mathbf{x}_{n}$ and velocities $\mathbf{v}_{n}$ follow the equations of motion [45]

$m \frac{d \mathbf{v}_{n}}{d t}=\mathbf{F}^{t}\left(\mathbf{x}_{n}\right)+\mathbf{F}_{n}^{C}\left(\left\{\mathbf{x}_{k}\right\}\right)+\mathbf{F}^{o}\left(\mathbf{x}_{n}\right)+\mathbf{F}^{D}\left(\mathbf{v}_{n}\right)+\mathbf{F}_{n}^{s}(t)$.

Here, $\mathbf{F}^{t}$ is the force provided by the ion trap which is assumed to be cylindrically symmetric with harmonic trap frequencies $\nu_{r}$ and $\nu_{z}$ in the radial and the longitudinal directions, respectively. For simplicity, we neglect any rf modulated motion and ion rotation associated with $\mathrm{rf}$ [20] and Penning [15] trapping, respectively. $\mathbf{F}_{n}^{C}$ represents the Coulomb forces on ion $n$ from all other ions in the ensemble. $\mathbf{F}^{D}$ is the mean Doppler laser cooling force modeled as a linear three-dimensional friction force

$$
\mathbf{F}^{D}\left(\mathbf{v}_{n}\right)=-\hbar k^{2} s \mathbf{v}_{n}
$$

where $k$ is the wave number of the cooling laser and $s$ is the saturation of the ion transition driven by this laser [46]. The stochastic noise associated with the random scattering of photons from the cooled ions is [46]

$$
\mathbf{F}_{n}^{s}(t)=\sqrt{\hbar^{2} k^{2} s \Gamma} \mathbf{X}_{n}(t),
$$

where $\Gamma$ is the excited state decay rate and $\mathbf{X}_{n}(t)$ are stochastic noise terms of zero mean and with 
$\left\langle\mathbf{X}_{n}(t) \mathbf{X}_{m}\left(t^{\prime}\right)\right\rangle=\delta_{n m} \delta\left(t-t^{\prime}\right)$. Finally, $\mathbf{F}^{o}$ is the induced dipole force exerted on the ions by a standing-wave optical field which is generated by the interference of two far-off resonant light beams.

Due to the $N^{2}$ scaling of the number of Coulomb interaction terms, numerical simulations of Eq. (1) are slow, and typically last a few hours on a single CPU core for $10 \mathrm{~ms}$ of temporal evolution for 1000 ions. This is to be compared to ion numbers of order $10^{4}$ used in experiments and time scales of order seconds on which crystal structure transitions have been observed [40, 41]. It is consequently not feasible to simulate the experimental observations on such long timescales and in particular to observe the formation of crystals from hot ion ensembles through cooling. Instead, we follow the approach of Refs. [40, 41] and initialize the simulations with an idealized structure consisting of 1000 ions, where a finite core (typically 125 ions) is fixed in an ideal bcc or fcc structure and the remaining ions form a surrounding shell minimizing the harmonic and Coulomb energies. For such a structure the ion density is constant through-out the crystal, with a value given by [9]

$$
\rho=\frac{m \epsilon_{0}(2 \pi)^{2}}{e^{2}}\left(\nu_{z}^{2}+2 \nu_{r}^{2}\right) .
$$

Likewise, the outer boundary of the crystal is expected to be spheroidal in shape with an aspect ratio (longitudinal to radial extension) which only depends on the ratio of the trapping frequencies [47]. Such a state, as shown in Fig. 1(a), resembles the experimentally observed structures $[40,41]$. Based on this configuration, full MD simulations are then performed for up to $10 \mathrm{~ms}$ under the influence of the forces described in Eq. (1). The laser cooling parameters are chosen to yield a stationary temperature of $0.5 \mathrm{mK}$, equivalent to e.g. the Doppler cooling limit for $\mathrm{Ca}^{+}$ions on the $4 \mathrm{~S}_{1 / 2}-4 \mathrm{P}_{1 / 2}$ transition, and cooling times of a few $10 \mu \mathrm{s}$. As we are mainly interested in the evolution of the crystal structure, we define a figure of merit, $\delta r_{\mathrm{bcc}}\left(\delta r_{\mathrm{fcc}}\right)$, which is the root-meansquare (rms) deviation of a fit of the ion separations in a subdomain containing a single ion and its nearest 8 (12) neighbors to a bcc (fcc) crystal structure, averaged over the 64 central ions in the ensemble. Note that such fits do not distinguish between an fcc and a hexagonally close packed (hcp) structure. For convenience, we express $\delta r$ in units of the Wigner-Seitz radius defined as

$$
r_{W S}=\left(\frac{3}{4 \pi \rho}\right)^{1 / 3}
$$

where $\rho$ is the ion density. Numerically, we then identify fcc crystals by the conditions $\delta r_{\mathrm{fcc}}<\delta r_{\mathrm{bcc}}$ and $\delta r_{\mathrm{fcc}}<0.17 r_{W S}$, and likewise bcc crystals by $\delta r_{\mathrm{bcc}}<$ $\delta r_{\mathrm{fcc}}$ and $\delta r_{\mathrm{bcc}}<0.17 r_{W S}$. If for a given ion ensemble $\delta r>0.17 r_{W S}$ for fits to both fcc and bcc lattices, the crystal structure can no longer meaningfully be determined.
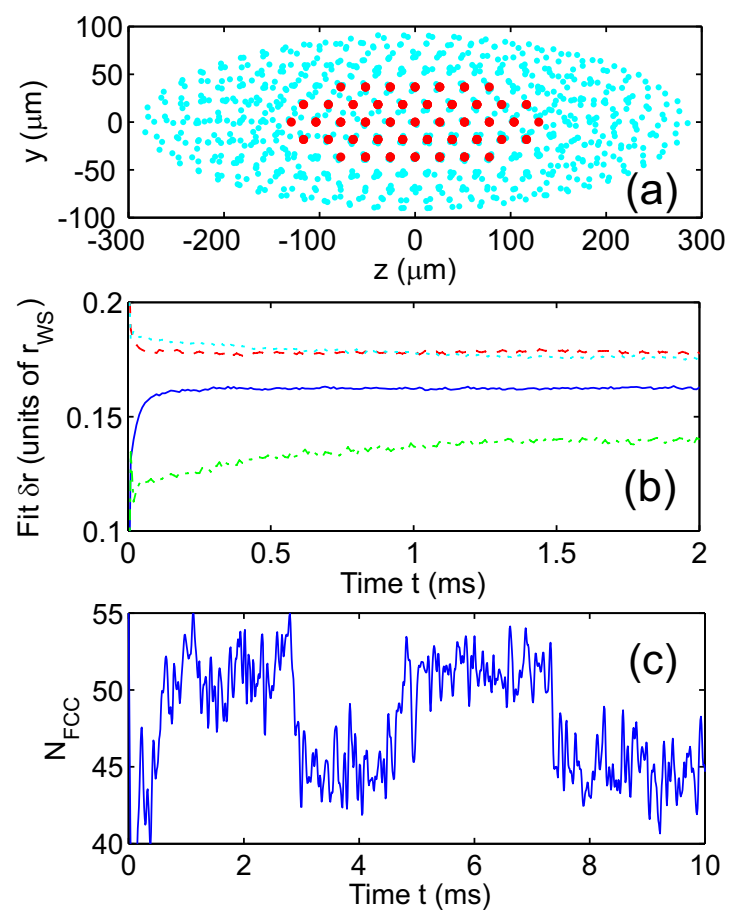

FIG. 1: (Color online) (a) Projection image of an ion Coulomb crystal composed of $N=1000$ ions in a trap with $\nu_{r}=$ $200 \mathrm{kHz}, \nu_{z}=100 \mathrm{kHz}$ with an fcc crystalline core of 125 ions (larger red dots). (b) Time evolution of the fits with fcc (solid line) and bcc (dashed) crystal structures for initialization with a fcc core, and fit with bcc (dash-dotted) and fcc (dotted) structures for a bcc initial core, without an optical potential. Average over 32 simulations. (c) Evolution of a single sample realization with initial fcc core.

Figure 1(b) shows the dynamics of $\delta r$ for the case without an induced dipole potential. The simulations are initiated with bcc and fcc structures in the central core, and with the [111] (bcc) and [011] (fcc) directions aligned along the trap $z$ axis, respectively. From perfect fits at time $t=0$, the structures quickly get slightly distorted, but during a simulation time of several ms they remain preferentially in the starting crystal structure. The fact that an initial fcc structure deteriorates much faster than the initial bcc structure is in line with the expectation that the bcc structure is the energetically more favorable. A single selected realization is depicted in Fig. 1(c). Here, we show the number of ions $N_{F C C}$ (out of the central 64 ions) where the rms deviation $\delta r$ of a fit with a local fcc structure is less than $0.17 r_{W S}$. This trajectory shows that structural transitions typically occur on short, $10 \mu$ s time scales (comparable to the trap oscillation and Doppler cooling times) in subdomains containing approximately 5-10 ions, a feature that is regularly found in these simulations and that has also been seen in experiments [41].

In the following we will investigate how this structural behavior of ion Coulomb crystals can be modified by 


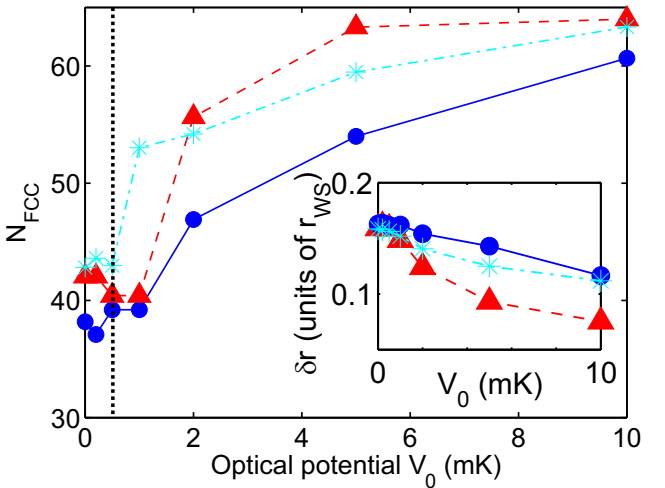

FIG. 2: (Color online) Average number of fcc ions (out of 64 core ions) after $10 \mathrm{~ms}$ of propagation versus dipole potential depths. The initial state has an ideal fcc core. Solid line and circles: trap as in Fig. 1, crystal aligned with [011] axis along $z, \Lambda=13.0 \mu \mathrm{m}$; dashed line and triangles: spherical trap with $\nu_{r}=\nu_{z}=150 \mathrm{kHz},[001]$ axis along $z, \Lambda=20.1 \mu \mathrm{m}$; dashdotted line and stars: spherical trap, [111] axis along $z, \Lambda=$ $23.2 \mu \mathrm{m}$. Inset: corresponding average $\mathrm{rms}$ fit deviation. In all cases, the stationary temperature is $0.5 \mathrm{mK}$ (dotted line).

adding a periodic induced dipole potential. Let us first consider a simple stationary standing wave with potential depth $V_{0}$ and periodicity $\Lambda$,

$$
V_{o}(x)=V_{0} \sin (\pi z / \Lambda)^{2} .
$$

Since for the same ion density fcc and bcc structures have incommensurable lattice planes, it is possible to choose $\Lambda$ such that only ions in either a bcc or an fcc crystal will fit perfectly into the minima of the potential (6). This should then stabilize ion crystals for extended periods of time. Results of simulations similar to those of Fig. 1(b) with an initial fcc-core ion crystal are shown in Fig. 2 as a function of the potential depth $V_{0}$ for three different configurations: an asymmetric trap with the potential addressing the (011) planes of the crystal, and a symmetric trap with the potential addressing either the $(001)$ or $(111)$ planes. For $V_{0}$ up to the stationary (Doppler) temperature of the ions $(\sim 0.5 \mathrm{mK})$, the potential does not significantly alter the dynamics of the ions and the crystal structure deteriorates as in Fig. 1(b). For $V_{0} \gtrsim 1 \mathrm{mK}$, however, the fidelity of maintaining the metastable fcc configuration improves significantly. The simulations with a spherical trap produce better stabilization, which we attribute mainly to reduced surface effects for this geometry.

Ideally, one would like to switch between different crystal structures. In principle, this can be achieved by switching the periodicity $\Lambda$ between two values favoring fcc and bcc crystals respectively. However, this approach is in general non-deterministic as the ions are required to follow a complex (random) walk to form the new crystal. In fact, numerical simulations showed that while this approach can switch the crystal structure locally it gen- (a)

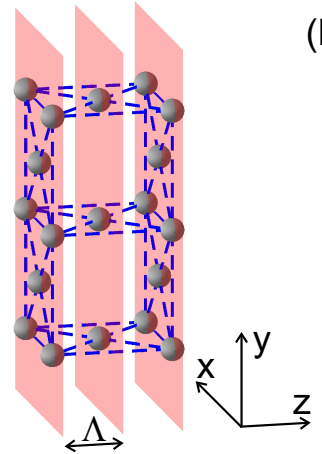

(b)

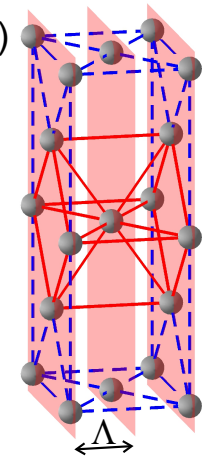

FIG. 3: (Color online) Schematic of crystal structure transformation from fcc to bcc along the Bain path using a fixed optical potential. (a) Two fcc unit cells (center face ions removed at front and at back for clarity). Shaded planes indicate standing wave potential minima. (b) After a uniform expansion in the transverse directions (density reduced by a factor of 2) a bcc unit cell is obtained (red solid lines).

erally destroys long range order.

A much better way to switch the ion crystal structure between fcc and bcc lattices would involve a continuous deformation along the Bain path [48]. Using optical potentials in conjunction with a harmonic ion trap there are two ways in which the Bain path can be realized. First, one can hold the ion trap and thus the ion density, Eq. (4), fixed and continuously vary the periodicity $\Lambda$. While our simulations showed that this scheme is successful, a continuous change of the interference pattern of the light beams generating the induced dipole potential may be difficult to carry out in practice. Alternatively, $\Lambda$ can be kept constant while the trap frequencies $\nu_{r}, \nu_{z}$ are varied by changing the voltages of the trap electrodes. This latter scheme is much more appealing experimentally and will thus be discussed in detail in the following.

A schematic of the proposed crystal structure transfer along the Bain path from fcc to bcc is shown in Fig. 3. Initially, Fig. 3(a), an fcc crystal is aligned with its [001] axis along the $z$ trap axis. $\Lambda$ is set to half the unit cell length, thus all ions are trapped at optical potential minima. Next, the density of the crystal is slowly reduced by a factor of 2 . With the ion ensemble being prevented from expanding in the $z$ direction by the optical potential, the crystal structure expands uniformly in the $x$ and $y$ directions by a factor $\sqrt{2}$. The resulting structure, Fig. $3(\mathrm{~b})$, now exhibits perfect bcc symmetry.

For the simulations we start with an ion crystal containing a core of 125 ions in bcc (fcc) configuration in a spherical trap with $\nu_{r}=\nu_{z}=150 \mathrm{kHz}$. Following Eq. (4), the full ensemble of $N=1000$ ions thus has a radius of $158 \mu \mathrm{m}$. The optical potential wells are orthogonal to the $z$ axis, as in Eq. (6), but now we assume finite beam sizes of $100 \mu \mathrm{m}$ waist in the $z$ and $y$ directions such that the ions outside the core area are free to move to the energetically most favorable positions in all directions. The 

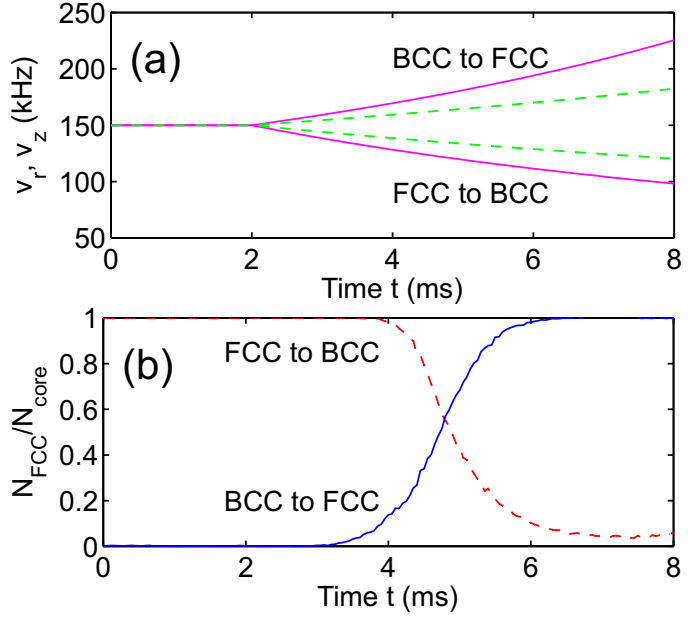

FIG. 4: (Color online) Continuous switching of ion Coulomb structures by varying the trap frequencies $\nu_{r}, \nu_{z}$ with a fixed optical potential $\left(V_{0}=10 \mathrm{mK}\right)$. (a) Time dependence of $\nu_{r}$ (solid) and $\nu_{z}$ (dashed) for switching of fcc to bcc (bottom curves) and bcc to fcc (top curves). (b) Corresponding fraction of core ions (out of 64) in a fcc configuration.

trap frequencies are kept constant for $2 \mathrm{~ms}$ and are then varied continuously between $t=2 \mathrm{~ms}$ and $8 \mathrm{~ms}$ as shown in Fig. 4(a). The time dependence of $\nu_{r}$ and $\nu_{z}$ is chosen such that the crystal density is decreased (increased) by a factor of 2 for the fcc to bcc (bcc to fcc) transition while the length of the ion crystal in $z$ direction is kept constant at all times [47].

The results of numerical simulations of this switching scenario are shown in Fig. 4(b). The simulations show a very efficient and fast transition of the crystal structure. After the switching sequence from fcc to bcc is finished, $5.7 \%$ of ions are in fcc and $89.3 \%$ in bcc configuration. The bcc to fcc transfer occurs with $99.9 \%$ efficiency. Thus, changing the trap potential allows for transfers between crystal structures along the Bain path with very high fidelity. Note that the two transfers shown here are not their respective inverse since both transfers start from a spherical geometry but the fcc-to-bcc transfer ends in a pan-cake shaped structure, the bcc-to-fcc transfer in a cigar-shape structure. Moreover, the final density is one half and twice the initial density, respectively. These differences in geometry may account for the different observed fidelities of the transfers.

Apart from crystal structure transitions along the Bain path we also investigated transitions along the Nishiyama-Wassermann and Kurdjumov-Sachs paths with optical potentials addressing the (111) planes of fcc and the (011) planes of bcc, respectively. While these transitions only involve a density change of $8 \%$, the paths required by the individual ions for structural transitions are much more complex than for the Bain path, involving contraction in one transverse direction, expansion in the other, and identical shearing motions of adjacent crystal planes (see, e.g., [49]). In MD simulations we observed no such correlated motion of all ions and thus a crystal structure transfer by a simple periodic potential (6) proved impossible. It should be noted, however, that stabilization of fcc and bcc structures is possible by these configurations, see Fig. 2.

The scenarios considered in the simulations above can experimentally be realized by applying a near resonant standing light field with the proper periodicity. In order to freely adjust the standing wave period for a given near resonant laser frequency, the crystal can be trapped in the region where two beams of a bow tie ring cavity cross [50]. Alternatively, a practically simpler solution may be to employ a linear optical cavity along the rf field free axis of a linear rf trap, as in recent experiments [26, 27 , and tune the ion density such that the periodicity of the relevant lattice planes of the fcc and bcc structures becomes an integer multiple of half the wavelength of the light field. We have checked by MD simulations that the transfer works as well in this scenario. However, due to a tremendous increase in computational time related to a much higher oscillation frequency for a given potential depth, extensive data have not been produced.

For estimating the typical laser power requirement for experimental realizations, we consider a linear cavity light field detuned by $5 \mathrm{THz}$ with respect to the $S_{1 / 2} \rightarrow P_{1 / 2}$ transition in ${ }^{40} \mathrm{Ca}^{+}$. Assuming a cavity mode waist diameter of $\sim 100 \mu \mathrm{m}$, a circulating power of about $10 \mathrm{~W}$ will be needed for producing optical potential depths of $10 \mathrm{mK}$. This power level is easily achievable by injecting a $\sim \mathrm{mW}$ field in a moderate finesse (a few thousand) cavity, and the corresponding photon scattering $\left(\sim 4 \times 10^{3} \mathrm{~s}^{-1}\right)$ and heating $(\sim 0.035 \mathrm{mK} / \mathrm{ms})$ rates can be neglected on the time scales considered.

As already mentioned, all of the results above were obtained assuming simple three-dimensional harmonic trapping potentials. Realistic Penning or linear rf ion traps have effective potentials of this type, yet their particular dynamical features introduce rotation and quadrupole perturbations, respectively. However, these motions have no components along the $z$ axis [11] and should therefore not limit the prospects of the schemes proposed here. Moreover, throughout the paper we have assumed the ions are cooled to the Doppler limit $(\sim 0.5 \mathrm{mK})$, but simulations have also shown similar results with ion temperatures of a few mK.

To summarize, the MD simulations of ion Coulomb crystals suggest that experimental investigations of structural transitions of such "clean" and low-density solids are feasible through the application of optical dipole forces. Crystalline effects investigated in such small-scale systems (several thousands of particles) are likely to help understand analogous effects of academic and technological interest within usual solid-state physics.

We acknowledge financial support from the EU (PICC STREP and CCQED ITN projects), the European Sci- 
ence Foundation (EuroQUAM program) and the U.K. Engineering and Physical Sciences Research Council (EP/E058949/1).

[1] E. L. Pollock and J. P. Hansen, Phys. Rev. A 8, 3110 (1973).

[2] D. J. Wineland, J. C. Bergquist, W. M. Itano, J. J. Bollinger, and C. H. Manney, Phys. Rev. Lett. 59, 2935 (1987).

[3] F. Diedrich, E. Peik, J. M. Chen, W. Quint, and H. Walther, Phys. Rev. Lett. 59, 2931 (1987).

[4] D. H. E. Dubin and T. M. O'Neil, Phys. Rev. Lett. 60, 511 (1988).

[5] R. W. Hasse and V. V. Avilov, Phys. Rev. A 44, 4506 (1991).

[6] H. Totsuji, T. Kishimoto, C. Totsuji, and K. Tsuruta, Phys. Rev. Lett. 88, 125002 (2002).

[7] R. W. Hasse and J. P. Schiffer, Ann. Phys. 203, 419 (1990).

[8] J. P. Schiffer, Phys. Rev. Lett. 70, 818 (1993).

[9] D. H. E. Dubin, Phys. Rev. E 53, 5268 (1996).

[10] T. Matthey, J. P. Hansen, and M. Drewsen, Phys. Rev. Lett. 91, 165001 (2003).

[11] J. P. Schiffer, M. Drewsen, J. S. Hangst, and L. Hornekær, Proc. Natl. Acad. Sci. 97, 10697 (2000).

[12] D. G. Enzer et al., Phys. Rev. Lett. 85, 2466 (2000).

[13] G. Morigi and S. Fishman, Phys. Rev. Lett. 93, 170602 (2004).

[14] A. Retzker et al., Phys. Rev. Lett. 101, 260504 (2008).

[15] T. B. Mitchell, J. J. Bollinger, D. H. E. Dubin, X.-P. Huang, W. M. Itano, and R. H. Baughman, Science 282, 1290 (1998).

[16] W. M. Itano et al., Science 279, 686 (1998).

[17] T. B. Mitchell, J. J. Bollinger, X.-P. Huang, and W. M. Itano, Opt. Express 2, 314 (1998).

[18] M. J. Jensen, T. Hasegawa, J. J. Bollinger, and D. H. E. Dubin, Phys. Rev. Lett. 94, 025001 (2005).

[19] G. Birkl, S. Kassner, and H. Walther, Nature 357, 310 (1992).

[20] M. Drewsen, C. Brodersen, L. Hornekær, J. S. Hangst, and J. P. Schiffer, Phys. Rev. Lett. 81, 2878 (1998).

[21] L. Hornekær, N. Kjærgaard, A. M. Thommesen, and M. Drewsen, Phys. Rev. Lett. 86, 1994 (2001).

[22] L. Hornekær and M. Drewsen, Phys. Rev. A 66, 013412 (2002).

[23] A. Mortensen, E. Nielsen, T. Matthey, and M. Drewsen, J. Phys. B: At. Mol. Opt. Phys. 40, F223 (2007).

[24] A. Dantan, J. P. Marler, M. Albert, D. Guénot, and M. Drewsen, Phys. Rev. Lett. 105, 103001 (2010).

[25] T. Rosenband et al., Science 319, 1808 (2008).
[26] P. F. Herskind, A. Dantan, J. P. Marler, M. Albert, and M. Drewsen, Nature Phys. 5, 494 (2009).

[27] M. Albert, A. Dantan, and M. Drewsen, Nature Photon. 5, 633 (2011)

[28] F. Dubin, C. Russo, H. G. Barros, A. Stute, C. Becher, P. O. Schmidt, and R. Blatt, Nature Phys. 6, 350 (2010).

[29] A. Kreuter, C. Becher, G. P. T. Lancaster, A. B. Mundt, C. Russo, H. Häffner, C. Roos, J. Eschner, F. SchmidtKaler, and R. Blatt, Phys. Rev. Lett. 92, 203002 (2004)

[30] M. Keller, B. Lange, K. Hayasaka, W. Lange, and H. Walther, Nature 431, 1075 (2004).

[31] P. Schindler, J. T. Barreiro, T. Monz, D. Nigg, M. Chwalla, M. Hennrich, and R. Blatt, Science 332, 1059 (2011).

[32] M. J. Biercuk, H. Uys, A. P. VanDevender, N. Shiga, W. M. Itano, and J. J. Bollinger, Nature 458, 996 (2009).

[33] J. P. Home, D. Hanneke, J. D. Jost, J. M. Amini, D. Leibfried, and D. J. Wineland, Science 325, 1227 (2009).

[34] K. Kim, M.-S. Chang, S. Korenblit, R. Islam, E. E. Edwards, J. K. Freericks, G.-D. Lin, L.-M. Duan, and C. Monrow, Nature 465, 590 (2010).

[35] J. W. Britton, B. C. Sawyer, A. C. Keith, C.-C. J. Wang, J. K. Freericks, M. J. Biercuk, and J. J. Bollinger, Nature 484, 489 (2012).

[36] K. Mølhave and M. Drewsen, Phys. Rev. A 62, 011401(R) (2000).

[37] P. F. Staanum, K. Højbjerre, P. S. Skyt, A. K. Hansen, and M. Drewsen, Nature Phys. 6, 271 (2010).

[38] T. Schneider, B. Roth, H. Duncker, I. Ernsting, and S. Schiller, Nature Phys. 6, 275 (2010).

[39] X. Tong, A. H. Winney, and S. Willitsch, Phys. Rev. Lett. 105, 143001 (2010).

[40] A. Mortensen, E. Nielsen, T. Matthey, and M. Drewsen, Phys. Rev. Lett. 96, 103001 (2006).

[41] M. Drewsen, T. Matthey, A. Mortensen, and J. P. Hansen, eprint arXiv:1202.2544v1 (2012).

[42] F. Wang and R. Ingalls, Phys. Rev. B 57, 5647 (1998).

[43] J. Wang, R. G. Hoagland, and A. Misra, J. Mater. Res. 23, 1009 (2008)

[44] R. Grimm, M. Weidemüller, and Y. B. Ovchinnikov, Adv. At. Mol. Opt. Phys. 42, 95 (2000).

[45] C. B. Zhang, D. Offenberg, B. Roth, M. A. Wilson, and S. Schiller, Phys. Rev. A 76, 012719 (2007).

[46] J. P. Gordon and A. Ashkin, Phys. Rev. A 21, 1606 (1980).

[47] L. Turner, Phys. Fluids 30, 3196 (1987).

[48] E. C. Bain, Trans. Am. Inst. Metall. Eng. 70, 25 (1924).

[49] L. Sandoval, H. M. Urbassek, and P. Entel, New J. Phys. 11, 103027 (2009).

[50] S. Bernon, T. Vanderbruggen, R. Kohlhaas, A. Bertoldi, A. Landragin, and P. Bouyer, New J. Phys. 13, 065021 (2011). 